\title{
Manasement Method of Planted Plants and Immigration Plants through Monitoring on Wildflower Garden - Case Study on Seoul Samgaksan Elementary School -
}

\author{
Yu-jin Song, Hye-young Jin, Chung-hee Lee, Yun-chang Jeon, and Chun-hee Nam* \\ Gardens and Education Division, Korea National Arboretum, South Korea
}

\begin{abstract}
This study was conducted to analyze the relationship between planted plants and immigration plants by monitoring the wildflower garden constructed at Seoul Samgaksan Elementary School. The results of this study are as follows:

First, plants planted in the garden 32 taxa, except for weakened only 1 taxa (Clematis patens), the remaining plants maintained or increased their populations. Among maintained Plants, that did not volume growth were 6 taxa and volume growth on the spot were 5 taxa. 15 taxa were spread around the planting area and 5 taxa spread sporadically to unpredictable areas other than the planting area. Although most of the planted plants show good growth, no special management is required, but Lonicera japonica needs the density management that cuts off the stem in the early spring and 5 sporadically spreading taxa, the planting intent of garden is attenuated, so remove the root when new leaves come out in spring.

Second, the total number of plants transferred after 2 years of wildflower garden constructed were 83 taxa total, which is 2.6 times that of planted plants. The seed disseminule form was analyzed as 52\% barochory, $27 \%$ anemochory\&hydrochory, $16 \%$ autochory and $5 \%$ zoochory. Also about $43 \%$ of the transferred plants were estimated to been imported from soil seed bank.

Third, we classified immigration plants into largely native plants and naturalized plants. The native plants were transferred to more than naturalized plants: 65 native plants, 1 cultivated plants and $17(22 \%)$ naturalized plants. The results of the monitoring of the rooftop garden 'Choroktteul' in Seoul City Hall showed that there were differences in the type and number of the immigration plants depending on the distance and quality of the surrounding greenery.

Fourth, the management method of immigration plants is divided into three categories: elimination, transplantation, and utilization. The 39 taxa were selected for the elimination: Invasive alien plants among naturalized plants, fast spreading plants, plants that expand sideways with rhizomes, and plants that interfere with the growth of planted plants: grow to over 1 meter in height and climbing plant. The elimination period should be dry, clear, windy day, remove the root without any residue. The transplanted plants are 18 taxa, tree, plants not suitable for habitat, and in elementary school textbooks. It is a group of 26 taxa which are used in the garden: for landscape that plants have ornamental values (flowers, leaves, and autumn colors etc.) but do not attenuate the intent of the garden, and for groundcover that plants have low plant height and fast spread. It is also possible to prevent immigration plants when you are mulching or planting plants densely populated areas when making a garden.
\end{abstract}

Key words: density management, plants management method, seed distribution type, quality of surrounding green space

\section{I . Introduction}

A garden is a space created by humans, not wild nature, and it continues to change its appearance yearly and seasonally. Gardens consist of plants that change over time as main materials, thus requiring continuous management. When gardens

Received on April 19, 2017. Revised on May 8, 2017.

Accepted on June 7, 2017.

*Comesponding author: eugenia@korea.kr

This study was supported by 'A study on the utilization of Korean

Native Plants for Garden culture expansion (Project Number: KNA12-22,14-6)'. are filled more with immigration plants than planted plants, their original forms and the intention of creation can be reduced. Some species of immigration plants, however, play a positive role in being a source of feed for wild birds and fish, and habitats, and resources for medicine, food or fertilizer, and preventing soil loss, and they also act as an important element for biodiversity. Therefore, it is necessary to manage immigration plants individually based on the accurate information on their species.

Studies on immigration plants can be divided into three groups. The first one is about immigration plants in natural 
spaces, including those on the distribution of naturalized plants and immigration plants in natural ecosystems, and those on monitoring resources in national parks. The second one is about immigration plants in planned spaces, and studies in the second group were conducted mainly in large-scale parks, such as Gildong Ecological Park (Kim et al., 2000), Yeouido Saetgang Ecological Park (Choi and Lee, 2001), and World Cup Park (Son, 2004). The third group is on the management of plants in gardens, including a study on the management methods of planted plants and invasive plants through monitoring a rooftop garden in Seoul City Hall (Choi et al., 2003), and a study on the characteristics of immigration plants (Jang et al., 2010).

Since the 'Act on the Creation and Furtherance of Arboretums and Gardens' was enacted in July, 2015, a series of garden expos were held in 'Korea, gaining attention from the public. At this point of time, it is necessary to conduct studies on methods of continuously maintaining gardens at a low cost with little management efforts in order to establish a gardening culture in daily life. It is necessary to remove immigration plants, viewing them as weeds, but it is also important to understand the characteristics of such plants and use them wisely, which changes gardens gradually into spaces harmonized with surrounding environments.

Against this backdrop, this study aims to monitor a wildflower garden in an elementary school in Seoul; analyze whether wild plants planted in the garden can grow well for a long time; examine immigration plants; and observe the relationship between planted plants and immigration plants. Based on the results, methods of managing plants in wildflower gardens created in urban areas are suggested in this study.

\section{Research Methods}

\section{Subjects}

This study was conducted at a wildflower garden (name as 'Wildflower Garden Feeling the Wind') created at Seoul Samgaksan Elementary School (141, Samyang-ro 19-gil, Gangbukgu, Seoul). The garden was constructed by the Korea National Arboretum within a large-scale apartment complex redeveloped in Samgaksan-dong (Mia 6 and 7 dongs), Gangbuk-gu in July,

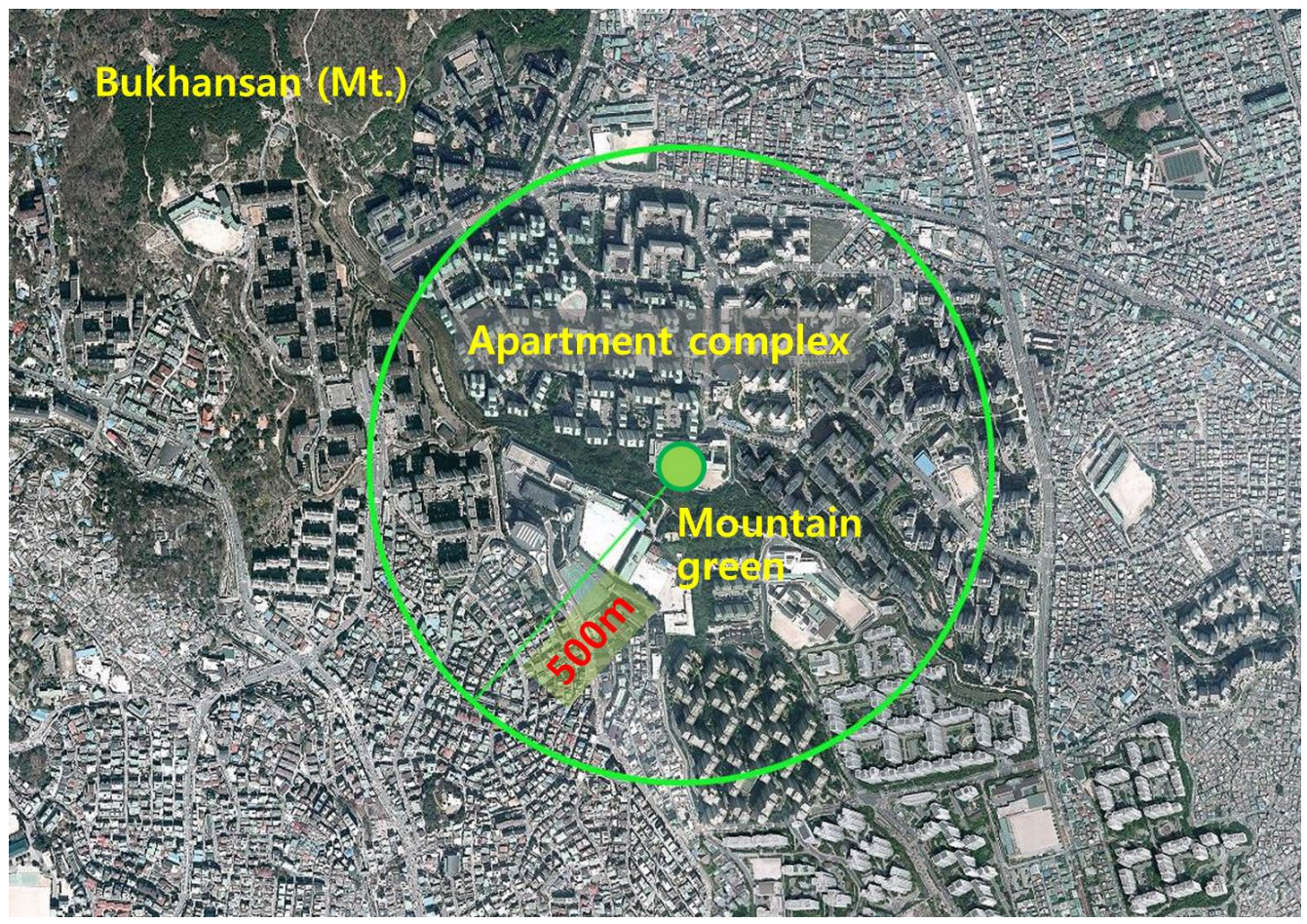

Fig. 1. Site around survey area and statue. 
Table 1. Seoul Samgaksan Elementary School wildflower garden planting plants statue.

\begin{tabular}{|c|c|c|}
\hline \multirow{2}{*}{$\begin{array}{l}\text { Family name } \\
\text { Pinaceae } \\
\text { 소나무과 }\end{array}$} & \multicolumn{2}{|c|}{ Scientific name / Korean Name } \\
\hline & Abies koreana Wilson & 구상나무 \\
\hline $\begin{array}{l}\text { Cornaceae } \\
\text { 층층나무과 }\end{array}$ & Cornus alba $\mathrm{L}$. & 흰말채나무 \\
\hline $\begin{array}{l}\text { Caprifoliaceae } \\
\text { 인동과 }\end{array}$ & Lonicera japonica Thunb. & 인동동굴 \\
\hline \multirow{5}{*}{$\begin{array}{l}\text { Ranunculacea } \\
\text { 미나리아재비과 }\end{array}$} & Clematis patens C.Morren \& Decne. & 큰꽃으아리 \\
\hline & $\begin{array}{l}\text { Adonis amurensis var. ramosa } \\
\text { Makino }\end{array}$ & 가지복수초 \\
\hline & $\begin{array}{l}\text { Aquilegia buergeriana var. } \\
\text { oxysepala (Trautv. \& Meyer) Kitam. }\end{array}$ & 매발톱 \\
\hline & Ranunculus japonicus Thunb. & 미나리아재비 \\
\hline & $\begin{array}{l}\text { Pulsatilla koreana (Yabe ex Nakai) } \\
\text { Nakai ex Mori }\end{array}$ & 할미꽃 \\
\hline $\begin{array}{l}\text { Juncaceae } \\
\text { 골풀과 }\end{array}$ & $\begin{array}{l}\text { Juncus effusus var. decipiens } \\
\text { Buchenau }\end{array}$ & 골풀 \\
\hline \multirow[b]{2}{*}{$\begin{array}{l}\text { Compositae } \\
\text { 국화과 }\end{array}$} & Aster maackii Regel & 좀개미취 \\
\hline & $\begin{array}{l}\text { Dendranthema zawadskii var. } \\
\text { tenuisectum Kitag. }\end{array}$ & 포천구절초 \\
\hline $\begin{array}{l}\text { Labiatae } \\
\text { 꿀풀과 }\end{array}$ & Thymus quinquecostatus Celak. & 백리향 \\
\hline $\begin{array}{l}\text { Crassulaceae } \\
\text { 돌나물과 }\end{array}$ & $\begin{array}{l}\text { Hylotelephium erythrostictum } \\
\text { (Miq.) H.Ohba }\end{array}$ & 꿩의비름 \\
\hline $\begin{array}{l}\text { Liliaceae } \\
\text { 백합과 }\end{array}$ & Hemerocallis fulva (L.) L. & 원추리 \\
\hline \multirow{2}{*}{$\begin{array}{l}\text { Saxifragaceae } \\
\text { 범의귀과 }\end{array}$} & $\begin{array}{l}\text { Astilbe rubra Hook.f. \& Thomas ex } \\
\text { Hook.f. }\end{array}$ & 노루오줌 \\
\hline & Mukdenia rossii (Oliv.) Koidz. & 돌단풍 \\
\hline $\begin{array}{l}\text { Gramineae } \\
\text { 벼과 }\end{array}$ & $\begin{array}{l}\text { Pennisetum alopecuroides (L.) } \\
\text { Spreng. }\end{array}$ & 수크령 \\
\hline $\begin{array}{l}\text { Lythraceae } \\
\text { 부처꽃과 }\end{array}$ & Lythrum salicaria $\mathrm{L}$. & 털부처꽃 \\
\hline $\begin{array}{l}\text { Iridaceae } \\
\text { 붓꽃과 }\end{array}$ & Iris setosa Pall. ex Link & 부채붓꽃 \\
\hline \multirow{5}{*}{$\begin{array}{l}\text { Cyperaceae } \\
\text { 사초과 }\end{array}$} & Carex japonica Thunb. & 개찌버리사초 \\
\hline & Carex aphanolepis Franch. \& Sav. & 골사초 \\
\hline & Carex lanceolata Boott & 그늘사초 \\
\hline & Carex boottiana Hook. \& Arn. & 밀사초 \\
\hline & Carex boottiana Hook. \& Arn. & 밀사초(무늬종) \\
\hline $\begin{array}{l}\text { Caryophyllaceae } \\
\text { 석죽과 }\end{array}$ & Arenaria juncea M.Bieb. & 벼룩이울타리 \\
\hline \multirow{2}{*}{$\begin{array}{l}\text { Primulaceae } \\
\text { 앵초과 }\end{array}$} & Primula sieboldii E.Morren & 앵초 \\
\hline & Lysimachia clethroides Duby & 큰까치수염 \\
\hline \multirow{2}{*}{$\begin{array}{l}\text { Rosaceae } \\
\text { 장미과 }\end{array}$} & Duchesnea indica (Andr.) Focke & 뱀딸기 \\
\hline & $\begin{array}{l}\text { Aruncus aethusifolius (H.Lév.) } \\
\text { Nakai }\end{array}$ & 한라개승마 \\
\hline $\begin{array}{l}\text { Campanulaceae } \\
\text { 초롱꽃과 }\end{array}$ & $\begin{array}{l}\text { Platycodon grandiflorum (Jacq.) } \\
\text { A.DC. }\end{array}$ & 도라지 \\
\hline $\begin{array}{l}\text { Scrophulariaceae } \\
\text { 현삼과 }\end{array}$ & Veronica linariifolia Pall. ex Link & 꼬리풀 \\
\hline $\begin{array}{l}\text { Fumariaceae } \\
\text { 현호색과 }\end{array}$ & Dicentra spectabilis (L.) Lem. & 금낭화 \\
\hline
\end{tabular}

2014. A fragmented green space at the foot of Bukhan Mountain is situated in the southern side of the elementary school, and the eastern, western and northern sides of the elementary school are surrounded by the apartment complex (Fig. 1). The playground of the school is located on the third floor of the building, and the floor is mostly made of concrete only with a very small green space. The elementary school shares its main gate with a middle school. The wildflower garden is created on the concrete floor $(10.3 \times 5.3 \times 0.45 \mathrm{~m})$ in an inner court surrounded by buildings, and the walls of the planting space were built with volcanic stone bricks. In the garden, 32 taxa1) of native plants ( 20 families, 28 genera) were planted (Table 1).

\section{Methods}

The wildflower garden was monitored in June and September, 2016, two years after the creation of the garden, when the growth and development of plants are most noticeable and all the weeds that grow in spring, summer and autumn can be observed. All the species of plants that were planted in the garden and other plants that were observed were surveyed, and their height and population, the coverage of planted plants and immigration plants, and their growth and development status (emergence, blooming, fruiting, seed disseminule form and withering) were recorded and photographed. In addition, to identify the route of introduction of immigration plants, plants that were observed within $500 \mathrm{~m}$ from the garden (flower beds within the school, residential areas, streets, surrounding mountain areas) were also surveyed (Fig. 1). The life forms and disseminule forms of immigration plants were also analyzed to collect their information. The surveyed plants were identified and classified based on Coloured Flora of Korea (Lee, 2003) and New Illustrations and Photographs of Naturalized Plants of Korea (Park, 2009), and listed according to the Korean Plant Names Index (Nature, 2017). The life forms and disseminule forms of the observed plants were rearranged based on Lineamenta Florae Koreae (Lee, 1996).

1) Taxa were not classified based on the existing classification scheme (Kingdom-Phylum-Class-Order-Family-Genus-Species), but listed by individual classification units (Operational Taxonomic Unit, OTU) that refer to the objects to be studied. These individual classification units may include species, subspecies, and variety. 


\section{Results and Discussion}

\section{Monitoring planted plants}

The 32 taxa of plants planted by the Korea National Arboretum in July, 2014 were all native plants that can grow in temperate regions in the middle part of Korea. In particular, considering the windy environment in the location, 6 grass taxa with high environmental adaptability (5 Cyperaceae species, 1 Gramineae species) were planted to ensure people feel the directions of wind like the main concept of the garden (Fig. 2).

The results of monitoring showed that most of the planted plants grew well, but Clematis patens C.Morren \& Decne. was weakened, indicating that the species failed to survive in the competition with Lonicera japonica Thunb., planted along the species. In addition, one of the five Abies koreana Wilson trees withered one year after planting, but the rest 4 trees grew well, which indicates that the tree withered due to other reasons than environmental conditions.

Most species maintained or increased their population, and those that maintained their populations but showed no growth in thickness were 6 taxa including Abies koreana Wilson, Hylotelephium erythrostictum (Miq.) H.Ohba, Iris setosa Pall. ex Link, Adonis amurensis var. ramosa Makino, Primula sieboldii E.Morren, and Dicentra spectabilis (L.) Lem. Those

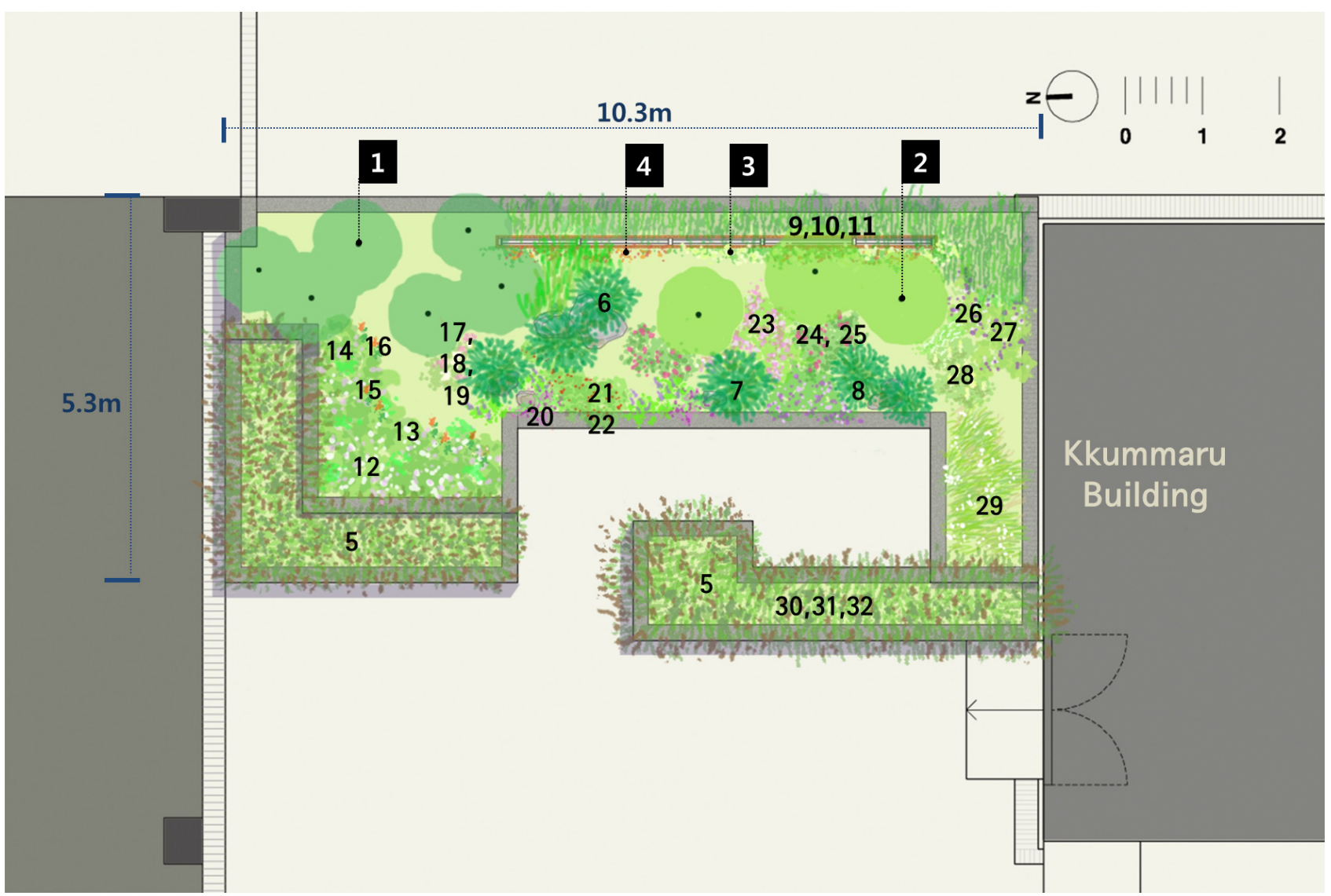

Fig. 2. Planting plan of wildflower garden.

1.구상나무 Abies koreana Wilson 2. 흰말채나무 Cornus alba L. 3. 인동덩굴 Lonicera japonica Thunb. 4. 큰꽃으아리 Clematis patens C.Morren \& Decne. 5. 수크령 Pennisetum alopecuroides (L.)Spreng. 6. 밀사초 Carex boottiana Hook. \& Arn. 7. 밀사초(무늬종) Carex boottiana Hook. \& Arn. 8. 개찌버리사초 Carex japonica Thunb. 9. 골풀 Juncus effusus var. decipiens Buchenau 10. 그늘사초 Carex lanceolata Boott 11. 골사초 Carex aphanolepis Franch. \& Sav. 12. 포천구절초 Dendranthema zawadskii var. tenuisectum Kitag. 13. 미나리아재비 Ranunculus japonicus Thunb. 14. 큰까치수염 Lysimachia clethroides Duby 15. 꼬리풀 Veronica linariifolia Pall. ex Link 16. 원추리 Hemerocallis fulva (L.) L. 17. 부채붓꽃 Iris setosa Pall. ex Link 18. 돌단풍 Mukdenia rossii (Oliv.) Koidz. 19. 좀개미취 Aster maackii Regel 20. 백리향 Thymus quinquecostatus Celak. 21. 가지복수초 Adonis amurensis var. ramosa Makino 22. 뱀딸기 Duchesnea indica (Andr.) Focke 23. 노루오줌 Astilbe rubra Hook.f. \& Thomas ex Hook.f. 24. 털부처꽃 Lythrum salicaria L. 25. 뀡의비름 Hylotelephium erythrostictum (Miq.) H.Ohba 26. 도라지 Platycodon grandiflorum (Jacq.) A.DC. 27. 금낭화 Dicentra spectabilis (L.) Lem. 28. 한라개승마 Aruncus aethusifolius (H.Lév.) Nakai 29. 벼룩이울타리 Arenaria juncea M.Bieb. 30. 매발톱 Aquilegia buergeriana var. oxysepala (Trautv. \& Meyer) Kitam. 31. 할미꽃 Pulsatilla koreana (Yabe ex Nakai) Nakai ex Mori 32. 앵초 Primula sieboldii E.Morren 
that maintained their population, and showed a growth in thickness were 5 taxa (Mukdenia rossii (Oliv.) Koidz, Duchesnea indica (Andr.) Focke, Carex lanceolata Boott, Carex boottiana Hook. \& Arn., and Carex boottiana Hook. \& Arn.), and among them, Mukdenia rossii (Oliv.) Koidz. and Cyperaceae species planted along the sides of native rocks grew in thickness only without increase in population. Those that showed increase in population were divided into two groups based on their dispersion patterns as follows: first, those that spread out near the area where they were originally planted, and second, those that were sporadically dispersed beyond the area. The first group included 15 taxa (Cornus alba L.; Lonicera japonica Thunb; Aquilegia buergeriana var. oxysepala (Trautv. \& Meyer) Kitam.; Ranunculus japonicus Thunb; Pulsatilla koreana (Yabe ex Nakai) Nakai ex Mori; Juncus effusus var. decipiens Buchenau; Thymus quinquecostatus Celak; Hemerocallis fulva (L.) L.; Pennisetum alopecuroides (L.) Spreng; Lythrum salicaria L.; Carex japonica Thunb.; Carex aphanolepis Franch. \& Sav.; Arenaria juncea M.Bieb.; Aruncus aethusifolius (H.Lév.) Nakai; Platycodon grandiflorum (Jacq.) A.DC.), and Lonicera japonica Thunb. covered almost all the wood fences, showing a noticeable growth and development. Cornus alba L., an arbor plant, extended their brachyblast. The second group included 5 taxa (Aster maackii Regel; Dendranthema zawadskii var. tenuisectum Kitag.; Astilbe rubra Hook.f. \& Thomas ex Hook.f.; Lysimachia clethroides Duby; Veronica linariifolia Pall. ex Link) (Table 2).

\section{Monitoring immigration plants}

The results of monitoring immigration plants are as follows. In June, 51 taxa, and in September, 67 taxa of immigration plants were observed, and among them, 35 taxa overlapped. The number of immigration plants 2 years after the creation of the garden was 83 taxa in total, 2.6 times the number of planted plants (32 taxa).

The life forms of immigration plants observed in this study include annual plants (37 taxa, including winter annual plants), biennial plants ( 7 taxa), and perennial plants (33 taxa). Most of them were herbaceous plants, but 6 taxa of arbor plants were also observed (Fraxinus rhynchophylla Hance; Acer tataricum subsp. ginnala (Maxim.) Wesm.; Nandina domestica Thunb.; Zanthoxylum schinifolium Siebold \& Zucc.; Spiraea prunifolia f. simpliciflora Nakai; Celastrus orbiculatus Thunb.). Among the immigration plants observed in this study, Compositae species were observed most (20 taxa, 24\%), followed by Gramineae species (11 taxa, 13\%), and Cyperaceae (5 taxa, $6 \%$ ) and Polygonaceae species (5 taxa, 6\%).

In terms of population, immigration plants were reclassified into 4 types based on the disseminule forms as follows: anemochory \& hydrochory, zoochory, autochory, and barochory (Lee, 1996). Those that showed two disseminule forms were recorded twice. Those of the barochory type accounted for $52 \%$, followed by the anemochory \& hydrochory type (27\%), the autochory type (16\%), and the zoochory type (5\%) (Table $3)$. Weedy immigration plants can survive for a long time under

Table 2. Wildflower garden planting plants monitoring results.

\begin{tabular}{|c|c|c|}
\hline $\begin{array}{l}\text { Num. of } \\
\text { plants }\end{array}$ & Degree & Scientific name / Korean Name \\
\hline Decrease & Weakness & Clematis patens C.Morren \& Decne. 큰꽃으아리 \\
\hline \multirow{2}{*}{ Maintain } & $\begin{array}{l}\text { Maintain } \\
\text { volume }\end{array}$ & $\begin{array}{l}\text { Abies koreana Wilson 구상나무, Hylotelephium erythrostictum (Miq.) H.Ohba 뀡의비름, Iris setosa Pall. ex Link 부채 } \\
\text { 붓꽃, Adonis ramosa Franchet 가지복수초, Primula sieboldii E.Morren 앵초, Dicentra spectabilis (L.) Lem. 금낭화 }\end{array}$ \\
\hline & $\begin{array}{l}\text { Volume } \\
\text { length growth }\end{array}$ & $\begin{array}{l}\text { Mukdenia rossii (Oliv.) Koidz. 돌단풍, Duchesnea indica (Andr.) Focke 뱀딸기, Carex lanceolata Boott 그늘사초, } \\
\text { Carex boottiana Hook. \& Arn. 밀사초, Carex boottiana Hook. \& Arn. 밀사초(무늬종) }\end{array}$ \\
\hline \multirow[t]{2}{*}{ Increase } & $\begin{array}{l}\text { Spread } \\
\text { out near }\end{array}$ & $\begin{array}{l}\text { Cornus alba L. 흰말채나무, Lonicera japonica Thunb. 인동덩굴, Aquilegia buergeriana var. oxysepala (Trautv. \& } \\
\text { Meyer) Kitam. 매발톱, Ranunculus japonicus Thunb. 미나리아재비, Pulsatilla koreana (Yabe ex Nakai) Nakai ex } \\
\text { Mori 할미꽃, Juncus effusus var. decipiens Buchenau 골풀, Thymus quinquecostatus Celak. 백리향, Hemerocallis } \\
\text { fulva (L.) L. 원추리, Pennisetum alopecuroides (L.) Spreng. 수크령, Lythrum salicaria L. 털부처꽃, Carex japonica } \\
\text { Thunb. 개찌버리사초, Carex aphanolepis Franch. \& Sav. 골사초, Arenaria juncea M.Bieb. 벼룩이울타리, Aruncus } \\
\text { aethusifolius (H.Lév.) Nakai 한라개승마, Platycodon grandiflorum (Jacq.) A.DC. 도라지 }\end{array}$ \\
\hline & $\begin{array}{l}\text { Sporadically } \\
\text { dispersed }\end{array}$ & $\begin{array}{l}\text { Aster maackii Regel 좀개미취, Dendranthema zawadskii var. tenuisectum Kitag. 포천구절초, Astilbe rubra Hook.f. } \\
\text { \& Thomas ex Hook.f. 노루오줌, Lysimachia clethroides Duby 큰까치수염, Veronica linariifolia Pall. ex Link 꼬리풀 }\end{array}$ \\
\hline
\end{tabular}


Table 3. Wildflower garden immigration plants statue and analysis.

\begin{tabular}{|c|c|c|c|c|c|c|c|c|c|}
\hline \multirow[b]{2}{*}{ Life form } & \multirow{2}{*}{\multicolumn{2}{|c|}{ Scientific name / Korean Name }} & \multirow[b]{2}{*}{$\begin{array}{l}\text { Naturalized } \\
\text { plants }\end{array}$} & \multirow[b]{2}{*}{$\begin{array}{l}\text { Num. of } \\
\text { plants }\end{array}$} & \multirow[b]{2}{*}{ Disseminule form } & \multicolumn{4}{|c|}{ Originate from immigration plants } \\
\hline & & & & & & $\begin{array}{l}\text { Flower bed } \\
\text { within the } \\
\text { school }\end{array}$ & $\begin{array}{l}\text { Residential } \\
\text { area \& } \\
\text { Streets }\end{array}$ & $\begin{array}{l}\text { Surrounding } \\
\text { mountain } \\
\text { areas }\end{array}$ & $\begin{array}{c}\text { Soil seed } \\
\text { bank } \\
\text { (assumption) }\end{array}$ \\
\hline \multirow[b]{2}{*}{ Tall tree } & Fraxinus rhynchophylla Hance & 물푸레나무 & & 1 & anemochory \& hydrochory & & & & 0 \\
\hline & $\begin{array}{l}\text { Acer tataricum subsp. ginnala } \\
\text { (Maxim.) Wesm. }\end{array}$ & 신나무 & & $2-5$ & anemochory \& hydrochory & $\bigcirc$ & & & \\
\hline \multirow{3}{*}{ Shrub } & Nandina domestica Thunb. & 남천 & & 1 & barochory & 0 & & & \\
\hline & $\begin{array}{l}\text { Zanthoxylum schinifolium Siebold \& } \\
\text { Zucc. }\end{array}$ & 산초나무 & & 1 & barochory & & & & $\bigcirc$ \\
\hline & $\begin{array}{l}\text { Spiraea prunifolia f. simpliciflora } \\
\text { Nakai }\end{array}$ & 조팝나무 & & $2-5$ & barochory & & & $\bigcirc$ & $\bigcirc$ \\
\hline $\begin{array}{l}\text { Climbing } \\
\text { tree }\end{array}$ & Celastrus orbiculatus Thunb. & 노박덩굴 & & $2-5$ & zoochory, barochory & $\bigcirc$ & & & \\
\hline \multirow{33}{*}{$\begin{array}{l}\text { Annual } \\
\text { plants }\end{array}$} & Setaria viridis (L.) P.Beauv. & 강아지풀 & & $21-50$ & barochory & $\bigcirc$ & $\bigcirc$ & & \\
\hline & Rorippa indica $($ L.) Hiern & 개갓냉이 & & $2-5$ & autochory & 0 & & & \\
\hline & Panicum bisulcatum Thunb. & 개기장 & & $2-5$ & barochory & & & & $\bigcirc$ \\
\hline & Erigeron annuus (L.) Pers. & 개망초 & $\bigcirc$ & $21-50$ & anemochory \& hydrochory & $\bigcirc$ & $\bigcirc$ & & \\
\hline & Persicaria longiseta (Bruijn) Kitag. & 개여뀌 & & $21-50$ & barochory & $\bigcirc$ & $\bigcirc$ & $\bigcirc$ & \\
\hline & $\begin{array}{c}\text { Persicaria thunbergii } \\
\text { (Siebold \& Zucc.) H.Gross ex Nakai }\end{array}$ & 고마리 & & $2-5$ & $\begin{array}{l}\text { barochory, anemochory \& } \\
\text { hydrochory }\end{array}$ & & & & O \\
\hline & Cyperus microiria Steud. & 금방동사니 & & $11-20$ & barochory & & & & $\bigcirc$ \\
\hline & Acalypha australis $\mathrm{L}$ & 깨풀 & & $21-50$ & zoochory, autochory & 0 & $\bigcirc$ & & \\
\hline & $\begin{array}{l}\text { Microstegium vimineum (Trin.) } \\
\text { A.Camus }\end{array}$ & 나도바랭이새 & & $\begin{array}{c}\text { more than } \\
51\end{array}$ & barochory & & & $\bigcirc$ & $\bigcirc$ \\
\hline & Commelina communis $\mathrm{L}$. & 닭의장풀 & & $11-20$ & barochory & $\bigcirc$ & & 0 & \\
\hline & Echinochloa crusgalli (L.) P.Beauv. & 돌피 & & $6-10$ & $\begin{array}{c}\text { barochory, anemochory \& } \\
\text { hydrochory }\end{array}$ & $\bigcirc$ & & $\bigcirc$ & \\
\hline & Conyza canadensis (L.) Cronquist & 망초 & 0 & $21-50$ & anemochory \& hydrochory & 0 & 0 & & \\
\hline & Pilea mongolica Wedd. 모시물통이 & & & $11-20$ & barochory & & & $\bigcirc$ & 0 \\
\hline & Digitaria violascens Link & 민바랭이 & & $21-50$ & barochory & $\bigcirc$ & $\bigcirc$ & & \\
\hline & Digitaria ciliaris (Retz.) Koel. & 바랭이 & & $21-50$ & barochory & & 0 & & \\
\hline & $\begin{array}{l}\text { Stellaria alsine var. undulata } \\
\text { (Thunb.) Ohwi }\end{array}$ & 벼룩나물 & & $21-50$ & barochory & & & & $\bigcirc$ \\
\hline & Erechtites hieracifolia Raf. & 붉은서나물 & $\bigcirc$ & $\begin{array}{c}\text { more than } \\
51\end{array}$ & anemochory \& hydrochory & $\bigcirc$ & 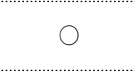 & & \\
\hline & Youngia japonica (L.) DC. & 뽀리뱅이 & & $21-50$ & anemochory \& hydrochory & & $\bigcirc$ & & \\
\hline & Fatoua villosa (Thunb.) Nakai & 뽕모시풀 & & $11-20$ & autochory & $\bigcirc$ & & & \\
\hline & $\begin{array}{l}\text { Persicaria nepalensis (Meisn.) } \\
\text { H.Gross }\end{array}$ & 산여뀌 & & $6-10$ & barochory & & & & $\bigcirc$ \\
\hline & Poa annua $\mathrm{L}$ & 새포아풀 & & $21-50$ & barochory & & & & $\bigcirc$ \\
\hline & Mollugo pentaphylla $\mathrm{L}$. & 석류풀 & & $21-50$ & barochory & & $\bigcirc$ & & \\
\hline & Veronica arvensis L. & 선개불알풀 & 0 & $11-20$ & barochory & & & & $\bigcirc$ \\
\hline & Euphorbia supina Raf. & 애기땅빈대 & 0 & $21-50$ & autochory & & $\bigcirc$ & & \\
\hline & $\begin{array}{l}\text { Phyllanthus ussuriensis Rupr. } \\
\text { \& Maxim. }\end{array}$ & 여우주머니 & & $11-20$ & autochory & & & & O \\
\hline & $\begin{array}{l}\text { Crepidiastrum denticulatum (Houtt.) } \\
\text { J.H.Pak \& Kawano }\end{array}$ & 이고들빼기 & & $6-10$ & anemochory \& hydrochory & & & $\stackrel{\bigcirc}{\text { (assumption) }}$ & \\
\hline & $\begin{array}{l}\text { Persicaria posumbu var. laxiflora } \\
\text { (Meisn.) H.Hara }\end{array}$ & 장대여뀌 & & $11-20$ & barochory & 0 & & & \\
\hline & Mazus pumilus (Burm.f.) Steenis & 주름잎 & & $11-20$ & barochory & $\bigcirc$ & & & \\
\hline & Centipeda minima (L.) A.Br. \& Asch. & 중대가리풀 & & $21-50$ & barochory & 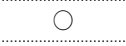 & $\bigcirc$ & & \\
\hline & $\begin{array}{c}\text { Mosla dianthera } \\
\text { (Buch.-Ham. ex Roxb.) Maxim. }\end{array}$ & 쥐깨풀 & & $\begin{array}{c}\text { more than } \\
51\end{array}$ & $\begin{array}{l}\text { barochory, anemochory \& } \\
\text { hydrochory }\end{array}$ & & & & O \\
\hline & Cyperus iria $\mathrm{L}$ & 참방동사니 & & $11-20$ & barochory & & & & $\bigcirc$ \\
\hline & $\begin{array}{l}\text { Aster subulatus var. sandwicensis } \\
\text { A.G.Jones }\end{array}$ & 큰비짜루국화 & O & 1 & anemochory \& hydrochory & & $\stackrel{\bigcirc}{\text { (assumption) }}$ & & \\
\hline & Galinsoga ciliata (Raf.) S.F.Blake & 털별꽃아재비 & 0 & $2-5$ & anemochory \& hydrochory & 0 & & $\bigcirc$ & \\
\hline
\end{tabular}


Table 3. (Continued).

\begin{tabular}{|c|c|c|c|c|c|c|c|c|c|}
\hline \multirow[b]{2}{*}{ Life form } & \multirow{2}{*}{\multicolumn{2}{|c|}{ Scientific name / Korean Name }} & \multirow[b]{2}{*}{$\begin{array}{l}\text { Naturalized } \\
\text { plants }\end{array}$} & \multirow[b]{2}{*}{$\begin{array}{l}\text { Num. of } \\
\text { plants }\end{array}$} & \multirow[b]{2}{*}{ Disseminule form } & \multicolumn{4}{|c|}{ Originate from immigration plants } \\
\hline & & & & & & $\begin{array}{c}\text { Flower bed } \\
\text { within the } \\
\text { school }\end{array}$ & $\begin{array}{l}\text { Residential } \\
\text { area \& } \\
\text { Streets } \\
\end{array}$ & $\begin{array}{c}\text { Surrounding } \\
\text { mountain } \\
\text { areas }\end{array}$ & $\begin{array}{c}\text { Soil seed } \\
\text { bank } \\
\text { (assumption) }\end{array}$ \\
\hline \multirow{7}{*}{$\begin{array}{l}\text { Biennial } \\
\text { plants }\end{array}$} & Agrostis clavata var. nukabo Ohwi & 겨이삭 & & $11-20$ & barochory & & $\bigcirc$ & & \\
\hline & $\begin{array}{l}\text { Crepidiastrum sonchifolium (Bunge) } \\
\text { Pak \& Kawano }\end{array}$ & 고들빼기 & & $11-20$ & anemochory \& hydrochory & & $\bigcirc$ & $\bigcirc$ & \\
\hline & $\begin{array}{l}\text { Trigonotis peduncularis (Trevir.) } \\
\text { Benth. ex Hemsl. }\end{array}$ & 꽃마리 & & $21-50$ & barochory & $\bigcirc$ & & O & \\
\hline & Oenothera biennis L. & 달맞이꽃 & $\bigcirc$ & $2-5$ & $\begin{array}{l}\text { autochory, anemochory \& } \\
\text { hydrochory }\end{array}$ & & & $\bigcirc$ & $\bigcirc$ \\
\hline & Sonchus oleraceus L. & 방가지똥 & $\bigcirc$ & $11-20$ & anemochory \& hydrochory & $\bigcirc$ & & $\bigcirc$ & \\
\hline & Stellaria media (L.) Vill. & 별꽃 & & $11-20$ & barochory & & & & 0 \\
\hline & $\begin{array}{l}\text { Cerastium holosteoides var. } \\
\text { hallaisanense (Nakai) Mizush. }\end{array}$ & 점나도나물 & & $11-20$ & barochory & & (assumption) & & \\
\hline \multirow{31}{*}{$\begin{array}{l}\text { Perennial } \\
\text { plants }\end{array}$} & Setaria faberii Herrm. & 가을강아지풀 & & $21-50$ & barochory & & & & 0 \\
\hline & $\begin{array}{c}\text { Agropyron tsukushiense var. transiens } \\
\text { (Hack.) Ohwi }\end{array}$ & 개밀 & & $2-5$ & barochory & & & $\bigcirc$ & $\bigcirc$ \\
\hline & $\begin{array}{l}\text { Pseudostellaria heterophylla (Miq.) } \\
\text { Pax ex Pax \& Hoffm. }\end{array}$ & 개별꽃 & & $2-5$ & barochory & & & & $\bigcirc$ \\
\hline & Hypericum erectum Thunb. & 고추나물 & & $11-20$ & barochory & & & & $\bigcirc$ \\
\hline & Oxalis corniculata $\mathrm{L}$. & 괭이밥 & & $\begin{array}{l}\text { more than } \\
\quad 51\end{array}$ & autochory, zoochory & $\bigcirc$ & $\bigcirc$ & & \\
\hline & Carex neurocarpa Maxim. & 갱이사초 & & $2-5$ & barochory & & & & $\bigcirc$ \\
\hline & Ambrosia trifida $\mathrm{L}$. & 단풍잎돼지풀 & $\bigcirc$ & $6-10$ & zoochory, barochory & & & $\bigcirc$ & 0 \\
\hline & Sedum sarmentosum Bunge & 돌나물 & & $\begin{array}{l}\text { more than } \\
51\end{array}$ & barochory & & $\bigcirc$ & O & \\
\hline & Viola collina Besser & 둥근털제비꽃 & & $2-5$ & zoochory, autochory & & & & 0 \\
\hline & Aster pilosus Willd. & 미국쑥부쟁이 & $\bigcirc$ & $6-10$ & anemochory \& hydrochory & & O & $\bigcirc$ & \\
\hline & Pinellia ternata (Thunb.) Breitenb. & 반하 & & $2-5$ & barochory & & & & 0 \\
\hline & Erigeron philadelphicus L. & 봄망초 & $\bigcirc$ & $2-5$ & anemochory \& hydrochory & & (assumption) & & \\
\hline & Allium macrostemon Bunge & 산달래 & & $21-50$ & barochory & & & & 0 \\
\hline & Eupatorium rugosum Houtt. & 서양등골나물 & $\bigcirc$ & $\begin{array}{c}\text { more than } \\
51\end{array}$ & anemochory \& hydrochory & $\bigcirc$ & $\bigcirc$ & $\bigcirc$ & \\
\hline & Taraxacum officinale Weber & 서양민들레 & $\bigcirc$ & $21-50$ & anemochory \& hydrochory & $\bigcirc$ & $\bigcirc$ & & \\
\hline & $\begin{array}{l}\text { Ixeris strigosa (H.Lév. \& Vaniot) } \\
\text { J.H.Pak \& Kawano }\end{array}$ & 선씀바귀 & & $11-20$ & anemochory \& hydrochory & & $\bigcirc$ & & \\
\hline & Potentilla freyniana Bornm. & 세잎양지꽃 & & $5-10$ & barochory & & & & 0 \\
\hline & Equisetum arvense L. & 쇠뜨기 & & $11-20$ & anemochory \& hydrochory & & & $\bigcirc$ & \\
\hline & Artemisia princeps Pamp. & 쑥 & & $11-20$ & barochory & & $\bigcirc$ & O & \\
\hline & Aster yomena (Kitam.) Honda & 쑥부쟁이 & & $2-5$ & $\begin{array}{l}\text { anemochory \& hydrochory, } \\
\text { barochory }\end{array}$ & & & $\stackrel{\bigcirc}{\text { (assumption) }}$ & \\
\hline & Rumex acetosella $\mathrm{L}$. & 애기수영 & $\bigcirc$ & $\begin{array}{l}\text { more than } \\
51\end{array}$ & barochory & & $\stackrel{\bigcirc}{\text { (assumption) }}$ & & \\
\hline & Poa pratensis L. & 왕포아풀 & $\bigcirc$ & $11-20$ & barochory & & & O & 0 \\
\hline & Viola mandshurica W.Becker & 제비꽃 & & $11-20$ & autochory & & $\bigcirc$ & & \\
\hline & Ixeris stolonifera A.Gray & 좀씀바귀 & & 51 이상 & anemochory \& hydrochory & & & & 0 \\
\hline & $\begin{array}{l}\text { Lysimachia vulgaris var. davurica } \\
\text { (Ledeb.) R.Kunth }\end{array}$ & 좁쌀풀 & & $2-5$ & barochory & & & & $\bigcirc$ \\
\hline & Plantago asiatica $\mathrm{L}$ & 질경이 & & $11-20$ & zoochory, barochory & 0 & $\bigcirc$ & $\bigcirc$ & \\
\hline & Carex breviculmis R.Br. & 청사초 & & $11-20$ & barochory & & $\bigcirc$ & & \\
\hline & Viola verecunda A.Gray & 콩제비꽃 & & $2-5$ & zoochory, autochory & & & & 0 \\
\hline & Trifolium repens $\mathrm{L}$. & 토끼풀 & $\bigcirc$ & $21-50$ & barochory & & & $\bigcirc$ & 0 \\
\hline & Kyllinga brevifolia Rottb. & 파대가리 & & $6-10$ & barochory & & & & 0 \\
\hline & Viola lactiflora Nakai & 흰젖제비꽃 & & $2-5$ & zoochory, autochory & & $\bigcirc$ & & \\
\hline
\end{tabular}


Table 3. (Continued).

\begin{tabular}{|c|c|c|c|c|c|c|c|c|c|}
\hline \multirow[b]{2}{*}{ Life form } & \multirow{2}{*}{\multicolumn{2}{|c|}{ Scientific name / Korean Name }} & \multirow[b]{2}{*}{$\begin{array}{l}\text { Naturalized } \\
\text { plants }\end{array}$} & \multirow[b]{2}{*}{$\begin{array}{l}\text { Num. of } \\
\text { plants }\end{array}$} & \multirow[b]{2}{*}{ Disseminule form } & \multicolumn{4}{|c|}{ Originate from immigration plants } \\
\hline & & & & & & $\begin{array}{c}\text { Flower bed } \\
\text { within the } \\
\text { school }\end{array}$ & $\begin{array}{l}\text { Residential } \\
\text { area \& } \\
\text { Streets }\end{array}$ & $\begin{array}{c}\text { Surrounding } \\
\text { mountain } \\
\text { areas }\end{array}$ & $\begin{array}{c}\text { Soil seed } \\
\text { bank } \\
\text { (assumption) }\end{array}$ \\
\hline \multirow{4}{*}{$\begin{array}{l}\text { Biennial } \\
\text { climbing } \\
\text { plants }\end{array}$} & Glycine soja Siebold \& Zucc. & 돌콩 & & $2-5$ & autochory & & & & $\bigcirc$ \\
\hline & $\begin{array}{l}\text { Amphicarpaea bracteata subsp. } \\
\text { edgeworthii (Benth.) H.Ohashi }\end{array}$ & 새콩 & & $2-5$ & autochory & & & & ○ \\
\hline & $\begin{array}{l}\text { Vigna angularis var. nipponensis } \\
\text { (Ohwi) Ohwi \& H.Ohashi }\end{array}$ & 새팥 & & $2-5$ & autochory & & ○ & $\bigcirc$ & \\
\hline & Humulus japonicus Siebold \& Zucc. & 환삼덩굴 & & $2-5$ & autochory & & $\mathrm{O}$ & & \\
\hline \multirow{2}{*}{$\begin{array}{l}\text { Perennial } \\
\text { climbing } \\
\text { plants }\end{array}$} & Metaplexis japonica (Thunb.) Makino & 박주가리 & & $2-5$ & anemochory \& hydrochory & $\bigcirc$ & $\bigcirc$ & & \\
\hline & Dioscorea nipponica Makino & 부채마 & & $2-5$ & anemochory \& hydrochory & & & & O \\
\hline
\end{tabular}

the ground (Rural Development Administration National Institute of Agricultural Sciences, 2008), and most of the immigration plants observed in an artificially created area in early years are grown from buried seeds. Although many species of the barochory type were observed in this study, the share of those of the anemochory \& hydrochory type is expected to gradually increase over time as more species immigrated from surrounding areas (Jang et al., 2010).

To trace their route of introduction, plants that were observed within $500 \mathrm{~m}$ from the wildflower garden in the elementary school were surveyed, and the results show that out of 83 taxa of immigration plants, 49 taxa were observed near the garden as follows: 25 taxa in flower beds within the school; 28 taxa in residential areas and on streets; 22 taxa in surrounding mountain areas (including overlapping species). The rest 34 taxa were not observed near the garden. Out of the species of which immigration routes were not identified, 2 taxa seemed to be introduced from surrounding mountain areas, 4 taxa from residential areas and streets, and 28 taxa from buried seeds that were brought with planted plants considering the disseminule forms of seeds and the ecology of plants. Among the immigration plants observed in this study, 8 taxa that showed the barochory and hydrochory types were observed in surrounding mountain areas, but it is difficult to assume that they were introduced from there within a short period of time, like 2 years. Thus, they are expected to grow from buried seeds.

Immigration plants are largely grouped into native plants and naturalized plants, and in this study 65 taxa of native plants, 1 taxon of cultivated plant, and 17 taxa of naturalized plants $(22 \%)$ were observed, more indicating native plants immigrated than naturalized plants. Out of the 17 taxa of naturalized plants, the disseminule form of 11 taxa is the anemochory \& hydrochory type, and they, except Veronica arvensis L., were all distributed in surrounding areas, indicating a high possibility of immigration from surrounding areas. Aster subulatus var. sandwicensis A.G.Jones, Ambrosia trifida L., Aster pilosus Willd., and Eupatorium rugosum Houtt. showed naturalized degree 4 (distributed in a limited area, but with large population) or 5 (cosmopolitan species with large population). They are categorized into the $3^{\text {rd }}$ stage of immigration (from 1964 to present), and thus they need to be monitored continuously (Park, 2009).

The results of monitoring the rooftop garden 'Choroktteul' in Seoul City Hall showed that there were more naturalized plants than native plants (Choi et al., 2003). The rooftop garden is located in downtown and surrounded by high-rise buildings, and it was created on a rooftop in an open environment with 10 $\mathrm{cm}$ deep soil. There is a stream (Cheonggyecheon (Stream) restoration) within $500 \mathrm{~m}$ from the garden, and green spaces at the foot of Inwang Mountain are $800 \mathrm{~m}$ away from the garden. For these reasons, unlike the wildflower garden, the target of this study, more naturalized plants seemed to be observed in the study. In the target of this study, unlike the Choroktteul rooftop garden, many native plants were introduced which is attributable to the fact that a fragmented green space at the foot of Bukhan Mountain is located in the southern side of the garden. The results of the monitoring the wildflower garden 'Wild Flower Garden Starting from Landfill' located within Pyeonghwa Park in World Cup Park in Sangam-dong, Seoul showed that $38 \%$ of immigration plants were naturalized 
plants and $62 \%$, native plants. This indicates a possibility of using native plants that immigrated from other places as gardening materials in a garden created with native plants. In addition, in an study on the immigration plants in the Garden of the Korea National Arboretum (Nam et al., 2017), over $75 \%$ of immigration plants were found to be introduced from the Gwangneung forest located close to the garden, which shows that the number and population of immigration plants are greatly affected by the distance from and the quality of surrounding green spaces.

\section{Methods of managing plants in gardens}

1) Managing planted plants

As the results of monitoring planted plants show in Table 4, except Clematis patens C.Morren \& Decne. of which population decreased, most of the planted plants grew well, and thus they did not require any special management. However, in the case of those that grew rapidly and are sporadically dispersed beyond the areas where they were originally planted, their population density needs to be managed to prevent the reduction of the intention of gardens. Population density can be relatively easily controlled by taking action at a seedling stage in early years.

Clematis patens C.Morren \& Decne. was the only species of which population decreased, and planted along with Lonicera japonica Thunb. Some leaves of Lonicera japonica Thunb. remained green even in winter, and it can grow well in any environment including shade, dry lands, rock fields, and coastal reclaimed lands. Lonicera japonica Thunb. is so prolific that just one tree can turn a whole village into a forest of Lonicera japonica Thunb. (Choi, 1997), and thus it seemed to suppress the growth and development of Clematis patens C.Morren \& Decne. that was planted along with it. Runners of this excessively prolific plant grew on the ground and into the areas where Clematis patens C.Morren \& Decne. was planted, and the runners have to be cut out in early spring to control its population density and to ensure visitors to see flowers of both plants.

There were 5 taxa that were sporadically dispersed beyond the planted areas into unpredictable areas (Aster maackii Regel; Dendranthema zawadskii var. tenuisectum Kitag.; Astilbe rubra Hook.f. \& Thomas ex Hook.f.; Lysimachia clethroides Duby; Veronica linariifolia Pall. ex Link). It is important to pull such species up by the roots when their new leaves are out in spring. In the case of Compositae plants within the planted areas (Aster maackii Regel, Dendranthema zawadskii var. tenuisectum Kitag.), their stems or branch tips, that is, their growing points, should be cut out to let axillary buds and side shoots grow using a shoot-top-cutting technique. By doing so, planted spaces can be filled with luxuriant plants (Korea National Arboretum, 2015).

\section{2) Managing immigration plants}

Methods of managing immigration plants including nonnative plants can be divided, depending on management materials and means, into ecological, physical, biological and chemical management types (Rural Development Administration National Institute of Agricultural Sciences, 2008). In this study, physical management methods are suggested only.

In this study, three types of physical management methods for immigration plants are suggested as follows: elimination,

Table 4. Management method of wildflower garden's planting plants.

\begin{tabular}{|c|c|c|c|c|c|c|}
\hline Case & Scientific name / Korean & me & & lagement Method & Time & Note \\
\hline $\begin{array}{l}\text { Plants spread } \\
\text { out near }\end{array}$ & Lonicera japonica Thunb. & 인동덩굴 & Thinning & $\begin{array}{l}\text { Cut the stem creeping the } \\
\text { ground Cut the stem that } \\
\text { extends to Clematis patens } \\
\text { planting area }\end{array}$ & $\begin{array}{l}\text { Early spring. } \\
\text { (Early March) }\end{array}$ & \\
\hline \multirow{5}{*}{$\begin{array}{l}\text { Plants } \\
\text { sporadically } \\
\text { dispersed } \\
\text { beyond the } \\
\text { area }\end{array}$} & Aster maackii Regel & 좀개미취 & \multirow{5}{*}{$\begin{array}{c}\text { Pulling up } \\
\text { or } \\
\text { Transplanting }\end{array}$} & \multirow{5}{*}{$\begin{array}{l}\text { Plants that have spread to areas } \\
\text { outside the planting area Pulling } \\
\text { up the roots or Transplanting }\end{array}$} & \multirow{3}{*}{$\begin{array}{l}\text { Mid-March, } \\
\text { when new } \\
\text { leaves come out }\end{array}$} & \multirow{3}{*}{$\begin{array}{l}\text { Plants in the } \\
\text { planting area, } \\
\text { shoot-top-cutting }\end{array}$} \\
\hline & $\begin{array}{l}\text { Dendranthema zawadskii var. } \\
\text { tenuisectum Kitag. }\end{array}$ & 포천구절초 & & & & \\
\hline & Lysimachia clethroides Duby & 큰까치수염 & & & & \\
\hline & Astilbe rubra Hook.f. \& Thomson & 노루오줌 & & & \multirow{2}{*}{$\begin{array}{l}\text { May, When new } \\
\text { leaves come out }\end{array}$} & \\
\hline & Veronica linariifolia Pall. ex Link & 꼬리풀 & & & & \\
\hline
\end{tabular}


transplanting and utilization. Those that did not meet the original plans and intention of the garden should be eliminated or transplanted into other places, and those that did not reduce the intention and threat the ecosystem of the garden can be utilized in the garden.

Those that have to be eliminated include invasive alien plants among naturalized plants; fast spreading plants; plants that expand sideways with rhizomes; and plants taller than $1 \mathrm{~m}$ and climbing plants that interfere with the growth of planted plants. Invasive alien plants among naturalized plants are species that cause serious risk to ecosystem, etc., and they need to be controlled under 'the Act on the Conservation and Utilization of Biological Diversity'. In the wildflower garden in this study, 4 taxa were observed (Ambrosia trifida L.; Aster pilosus Willd.; Eupatorium rugosum Houtt.; Rumex acetosella L.) and they should be immediately removed as soon as they are found. Fast spreading plants include 19 taxa (Setaria viridis (L.) P.Beauv.; Panicum bisulcatum Thunb; Erigeron annuus (L.) Pers.; Acalypha australis L.; Microstegium vimineum (Trin.) A.Camus; Echinochloa crusgalli (L.) P.Beauv.; Conyza canadensis (L.) Cronquist; Digitaria violascens Link; Digitaria ciliaris (Retz.) Koel.; Erechtites hieracifolia Raf.; Veronica arvensis L.; Euphorbia supina Raf.; Aster subulatus var. sandwicensis A.G.Jones; Galinsoga ciliata (Raf.) S.F.Blake; Sonchus oleraceus L.; Setaria faberii Herrm.; Agropyron tsukushiense var. transiens (Hack.) Ohwi; Erigeron philadelphicus L.; Taraxacum officinale Weber). If they are not removed at a seedling stage, they spread exponentially, so that it becomes difficult to control their population. Therefore, they should be eliminated when they start to flower in spring. Plants that expand sideways with rhizomes include 5 taxa (Equisetum arvense L.; Artemisia princeps Pamp.; Rumex acetosella L.; Poa pratensis L.; Trifolium repens L.), and it is important to completely eliminate the plants including roots. In particular, Equisetum arvense $\mathrm{L}$. spreads and puts down root deep into the ground so quickly that it becomes very difficult to remove it later unless controlling at an early stage. Therefore, it should be immediately removed as soon as they are found before their sporangium cones disperse spores in early spring. Trifolium repens $\mathrm{L}$. is a familiar plant to people as it is introduced in elementary school textbooks. Some can be transplanted to use as a learning material for students, and the rest should be all removed. Since it expands sideways, it should be managed within a container to prevent its roots from going farther. Plants taller than $1 \mathrm{~m}$ (5 taxa including Panicum bisulcatum Thunb.; Echinochloa crusgalli (L.) P.Beauv.; Aster subulatus var. sandwicensis A.G.Jones; Sonchus oleraceus L.; Setaria faberii Herrm.) are indistinguishable from planted plants, and thus they should be eliminated at an early stage to maintain the intention of planting. Climbing plants (6 taxa including Glycine soja Siebold \& Zucc.; Amphicarpaea bracteata subsp. edgeworthii (Benth.) H.Ohashi; Vigna angularis var. nipponensis (Ohwi) Ohwi \& H.Ohashi; Humulus japonicus Siebold \& Zucc.; Metaplexis japonica (Thunb.) Makino; Dioscorea nipponica Makino) also interfere with the growth of planted plants, and should be removed. Dry, sunny and windy days, not rainy days are suitable for removing plants, because the wind can quickly evaporate moisture in weeds rooted out, and keep them from taking root in the ground again (Oh, 2009).

Transplanted plants are removed from the wildflower garden to maintain the original plan and intention of the garden, but they can be utilized elsewhere. They include tree plants, plants not suitable for the habitat, and plants introduced in elementary school textbooks. In the case of tree plants, they are likely to naturally die out from a long-term perspective, and also to grow taller than the capacity of the garden, and thus they need to be transplanted into other environments suitable for them at an early stage. Plants that are not suitable for the habitat of the garden were 2 taxa including Persicaria thunbergii (Siebold \& Zucc.) H.Gross ex Nakai, and Pilea mongolica Wedd. Since they grow in wet areas, they seem to grow from buried seeds. They can be utilized as scenic materials, and thus need to be transplanted into other environments suitable for their growth and development. Meanwhile, as the target of monitoring in this study is a garden in an elementary school, plants that are introduced in elementary school textbooks (Reu, 2016) can be used for educational purposes to increase the effectiveness of learning. In the case of Setaria viridis (L.) P.Beauv., as it spreads very quickly, it is necessary to pull it up by the roots after transplanting some for educational purposes.

Among immigration plants, those that can be maintained and utilized in the garden can be divided into landscaping and ground-covering types. Plants for landscaping should have ornamental values in their flowers, leaf colors and textures, 
Table 5. Management method of wildflower garden's immigration plants.

\begin{tabular}{|c|c|c|c|}
\hline Method & \multicolumn{2}{|c|}{ Type } & Scientific name / Korean Name \\
\hline \multirow{5}{*}{ Elimination } & \multicolumn{2}{|c|}{$\begin{array}{l}\text { Invasive alien plants among } \\
\text { naturalized plants }\end{array}$} & $\begin{array}{l}\text { Ambrosia trifida L. 단풍잎돼지풀, Aster pilosus Willd. 미국쑥부쟁이, Eupatorium rugosum Houtt. 서양등골 } \\
\text { 나물, Rumex acetosella L. 애기수영 }\end{array}$ \\
\hline & \multicolumn{2}{|c|}{ Fast spreading plants } & $\begin{array}{l}\text { Setaria viridis (L.) P.Beauv. 강아지풀, Panicum bisulcatum Thunb 개기장, Erigeron annuus (L.) Pers. 개망초, } \\
\text { Acalypha australis L. 깨풀, Microstegium vimineum (Trin.) A.Camus 나도바랭이새, Echinochloa crusgalli } \\
\text { (L.) P.Beauv. 돌피, Conyza canadensis (L.) Cronquist 망초, Digitaria violascens Link 민바랭이, Digitaria } \\
\text { ciliaris (Retz.) Koel. 바랭이, Erechtites hieracifolia Raf. 붉은서나물, Veronica arvensis L. 선개불알풀, } \\
\text { Euphorbia supina Raf. 애기땅빈대, Aster subulatus var. sandwicensis A.G.Jones 큰비짜루국화, Galinsoga } \\
\text { ciliata (Raf.) S.F.Blake 털별꽃아재비, Sonchus oleraceus L. 방가지똥, Setaria faberii Herrm. 가을강아지풀, } \\
\text { Agropyron tsukushiense var. transiens (Hack.) Ohwi 개밀, Erigeron philadelphicus L. 봄망초, Taraxacum } \\
\text { officinale Weber 서양민들레 }\end{array}$ \\
\hline & \multicolumn{2}{|c|}{$\begin{array}{l}\text { Plants that expand sideways with } \\
\text { rhizomes }\end{array}$} & $\begin{array}{l}\text { Equisetum arvense L. 쇠뜨기, Artemisia princeps Pamp. 쑥, Rumex acetosella L. 애기수영, Poa pratensis L. } \\
\text { 왕포아풀, Trifolium repens L. 토끼풀 }\end{array}$ \\
\hline & \multirow{2}{*}{$\begin{array}{l}\text { Plants that } \\
\text { interfere with } \\
\text { the growth of } \\
\text { planted plants }\end{array}$} & $\begin{array}{l}\text { Tall Plants } \\
\text { (more than } 1 \mathrm{~m} \text { ) }\end{array}$ & $\begin{array}{l}\text { Panicum bisulcatum Thunb. 개기장, Echinochloa crusgalli (L.) P.Beauv. 돌피, Aster subulatus var. } \\
\text { sandwicensis A.G.Jones 큰비짜루국화, Sonchus oleraceus L. 방가지똥, Setaria faberii Herrm. 가을강아지풀 }\end{array}$ \\
\hline & & Climbing plant & $\begin{array}{l}\text { Glycine soja Siebold \& Zucc. 돌콩, Amphicarpaea bracteata subsp. edgeworthii (Benth.) H.Ohashi 새콩, Vigna } \\
\text { angularis var. nipponensis (Ohwi) Ohwi \& H.Ohashi 새팥, Humulus japonicus Siebold \& Zucc. 환삼덩굴, } \\
\text { Metaplexis japonica (Thunb.) Makino 박주가리, Dioscorea nipponica Makino 부채마 }\end{array}$ \\
\hline \multirow{3}{*}{ Transplanting } & \multicolumn{2}{|c|}{ Tree } & $\begin{array}{l}\text { Fraxinus rhynchophylla Hance 물푸레나무, Acer tataricum subsp. ginnala (Maxim.) Wesm. 신나무, Nandina } \\
\text { domestica Thunb. 남천, Zanthoxylum schinifolium Siebold \& Zucc. 산초나무, Spiraea prunifolia f. } \\
\text { simpliciflora Nakai 조팝나무, Celastrus orbiculatus Thunb. 노박덩굴 }\end{array}$ \\
\hline & \multicolumn{2}{|c|}{ Plants not suitable for habitat } & Persicaria thunbergii (Siebold \& Zucc.) H.Gross ex Nakai 고마리, Pilea mongolica Wedd. 모시물통이 \\
\hline & \multicolumn{2}{|c|}{$\begin{array}{l}\text { Plants in elementary } \\
\text { school textbooks }\end{array}$} & $\begin{array}{l}\text { Spiraea prunifolia f. simpliciflora Nakai 조팝나무, Celastrus orbiculatus Thunb. 노박덩굴, Setaria viridis (L.) } \\
\text { P.Beauv. 강아지풀, Commelina communis L. 닭의장풀, Crepidiastrum sonchifolium (Bunge) Pak \& Kawano } \\
\text { 고들빼기, Oenothera biennis L. 달맞이꽃, Oxalis corniculata L. 괭이밥, Artemisia princeps Pamp. 쑥, Viola } \\
\text { mandshurica W.Becker 제비꽃, Trifolium repens L. 토끼풀 }\end{array}$ \\
\hline \multirow[t]{2}{*}{ Utilization } & \multicolumn{2}{|c|}{ For landscape } & $\begin{array}{l}\text { Rorippa indica (L.) Hiern 개갓냉이, Persicaria longiseta (Bruijn) Kitag. 개여뀌, Cyperus microiria Steud. 금 } \\
\text { 방동사니, Youngia japonica (L.) DC. 뽀리뱅이, Fatoua villosa (Thunb.) Nakai 뽕모시풀, Persicaria } \\
\text { nepalensis (Meisn.) H.Gross 산여뀌, Phyllanthus ussuriensis Rupr. \& Maxim. 여우주머니, Crepidiastrum } \\
\text { denticulatum (Houtt.) J.H.Pak \& Kawano 이고들빼기, Persicaria posumbu var. laxiflora (Meisn.) H.Hara 장대 } \\
\text { 여뀌, Mosla dianthera (Buch.-Ham. ex Roxb.) Maxim. 쥐깨풀, Cyperus iria L. 참방동사니, Hypericum } \\
\text { erectum Thunb. 고추나물, Carex neurocarpa Maxim. 괭이사초, Viola collina Besser 둥근털제비꽃, Pinellia } \\
\text { ternata (Thunb.) Breitenb. 반하, Ixeris strigosa (H.Lév. \& Vaniot) J.H.Pak \& Kawano 선씀바귀, Potentilla } \\
\text { freyniana Bornm. 세잎양지꽃, Aster yomena (Kitam.) Honda 쑥부쟁이, Lysimachia vulgaris var. davurica } \\
\text { (Ledeb.) R.Kunth 좁쌀풀, Viola verecunda A.Gray 콩제비꽃, Viola lactiflora Nakai 흰젖제비꽃 }\end{array}$ \\
\hline & \multicolumn{2}{|c|}{ For groundcover } & $\begin{array}{l}\text { Stellaria alsine var. undulata (Thunb.) Ohwi 벼룩나물, Poa annua L. 새포아풀, Mollugo pentaphylla L. 석류 } \\
\text { 풀, Mazus pumilus (Burm.f.) Steenis 주름잎, Centipeda minima (L.) A.Br. \& Asch. 중대가리풀, Agrostis } \\
\text { clavata var. nukabo Ohwi 겨이삭, Trigonotis peduncularis (Trevir.) Benth. ex Hemsl. 꽃마리, Stellaria media } \\
\text { (L.) Vill. 별꽃, Cerastium holosteoides var. hallaisanense (Nakai) Mizush. 점나도나물, Pseudostellaria } \\
\text { heterophylla (Miq.) Pax ex Pax \& Hoffm. 개별꽃, Sedum sarmentosum Bunge 돌나물, Allium macrostemon } \\
\text { Bunge 산달래, Ixeris stolonifera A.Gray 좀씀바귀, Plantago asiatica L. 질경이, Carex breviculmis R.Br. 청사 } \\
\text { 초, Kyllinga brevifolia Rottb. 파대가리 }\end{array}$ \\
\hline
\end{tabular}

and autumn leaf colors, and do not reduce the intention of gardens. In the wildflower garden monitored in this study, 21 taxa can be utilized for landscaping. Plants for ground-covering are usually of short height ( $20 \mathrm{~cm}$ or shorter), spread quickly and have shallow roots. In the wildflower garden, 16 taxa can be maintained and utilized (Table 5).

\section{Conclusions}

As gardens use plants as main materials, they continue to change their appearance yearly and seasonally. To maintain the original forms and intention of gardens, continuous management is essential. This study aimed to monitor a wildflower garden created at Seoul Samgaksan Elementary School, to observe the relationship between planted plants and immigration plants, and thus to suggest methods of managing plants, and the results of monitoring and the suggested methods are as follows. The characteristics of planted plants and immigration plants should be fully understood in other wildflower gardens created in urban areas, and based on the understanding, they should be managed by plant types.

First, among the 32 taxa of planted plants in the garden, most 
of them, except Clematis patens C.Morren \& Decne. that was weakened, maintained or increased their populations. Those that maintained their populations but showed no growth in thickness were 6 taxa (Abies koreana Wilson, Hylotelephium erythrostictum (Miq.) H.Ohba, Iris setosa Pall. ex Link, Adonis amurensis var. ramosa Makino, Primula sieboldii E.Morren, and Dicentra spectabilis (L.) Lem). Those that maintained their population, and showed a growth in thickness were 5 taxa (Mukdenia rossii (Oliv.) Koidz, Duchesnea indica (Andr.) Focke, Carex lanceolata Boott, Carex boottiana Hook. \& Arn., and Carex boottiana Hook. \& Arn.). Plants that spread out near the area where they were originally planted included 15 taxa (Cornus alba L.; Lonicera japonica Thunb; Aquilegia buergeriana var. oxysepala (Trautv. \& Meyer) Kitam.; Ranunculus japonicus Thunb; Pulsatilla koreana (Yabe ex Nakai) Nakai ex Mori; Juncus effusus var. decipiens Buchenau; Thymus quinquecostatus Celak; Hemerocallis fulva (L.) L.; Pennisetum alopecuroides (L.)Spreng; Lythrum salicaria L.; Carex japonica Thunb.; Carex aphanolepis Franch. \& Sav.; Arenaria juncea M.Bieb.; Aruncus aethusifolius (H.Lév.) Nakai; Platycodon grandiflorum (Jacq.) A.DC.). Plants that were sporadically dispersed into unpredictable areas beyond the area included 5 taxa (Aster maackii Regel; Dendranthema zawadskii var. tenuisectum Kitag.; Astilbe rubra Hook.f. \& Thomas ex Hook.f.; Lysimachia clethroides Duby; Veronica linariifolia Pall. ex Link). Most of the planted plants have grown well, and thus they do not require any special management. However, it is necessary to cut out runners of Lonicera japonica Thunb. in early spring for density management. As the 5 taxa that are sporadically dispersed beyond the planted areas can reduce the intention of the garden, it is necessary to pull them out by the roots and be transplanted when their new leaves are out in spring.

Second, the number of immigration plants 2 years after the creation of the garden was 83 taxa in total, 2.6 times the number of planted plants. In terms of disseminule forms, those of the barochory type accounted for $52 \%$, followed by the anemochory $\&$ hydrochory type $27 \%$, the autochory type $16 \%$, and the zoochory type $5 \%$. Approximately $43 \%$ of the immigration plants seemed to grow from buried seeds, and thus it is important to consider that other seeds in soil can be brought with planted plants when managing gardens.
Third, immigration plants are largely grouped into native plants and naturalized plants, and in this study 65 taxa of native plants, 1 taxon of cultivated plant, and 17 taxa of naturalized plants (22\%) were observed, indicating native plants immigrated more than naturalized plants. The results are inconsistent with those of monitoring the rooftop garden 'Choroktteul' in Seoul City Hall where naturalized plants were observed more. This indicates that the number and population of immigration plants are affected by the distance from and the quality of surrounding green spaces.

Fourth, in this study, three types (elimination, transplanting and utilization) of management methods for immigration plants were suggested. Plants that should be eliminated were 39 taxa, including invasive alien plants among naturalized plants; fast spreading plants; plants that expand sideways with rhizomes; and plants taller than $1 \mathrm{~m}$ and climbing plants that interfere with the growth of planted plants. It is important to pull them out by the roots on dry, sunny and windy days, not rainy days at a seedling stage. Plants that should be transplanted were 18 taxa including tree plants, plants not suitable for the habitat, and plants introduced in elementary school textbooks. Plants that can be maintained and utilized in the garden were 26 taxa, including those for landscaping that have ornamental values in their flowers, leaf colors and textures, and autumn leaf colors, and do not reduce the intention of gardens, and those for ground-covering that have short height, spread quickly and have shallow roots. When creating a garden, immigration plants can be also prevented by mulching plants with barks, wood chips, straw, and coarse decomposed granite or gravels, or covering the land densely with ground-covering plants without any vacant space. In this study, physical methods of management were suggested only. As species in the wildflower garden are diversified over time, it will be necessary to develop biological management methods that use interactions between living organisms such as microorganisms, insects and allelopathy.

As this study monitored planted plants and immigration plants 2 years after the creation of the wildflower garden and suggested methods of managing the garden at an early stage only, it will be necessary to continue to monitor plants and suggest measures to manage the wildflower garden by stages. 


\section{References}

Choi, B.E. and K.J. Lee. 2001. The management plant of the Youido Satkang ecology park based on ecosystem monitoring. Kor. J. Env. Eco. 15(1):26-38.

Choi, H.S., S.Y. Hong, K.G. Kim, B.E. Yang, and W.Y. Oh. 2003. A study of management method of planted plants and invasive plants through monitoring on rooftop garden "Choroktteul" in Seoul city hall.J. Korean Inst. Landsc. Archit. 31(3):114-124.

Choi, M.S. 1997. Lonicera japonica. Landsc. tree. 40(9):25-26.

Jang, J., C.H. Oh, S.H. Yoon, and H.Y. Oh. 2010, April. The Research of immigration plants characteristics in the rooftop garden of Seoul city hall. Korean Soc. Environ. Ecol., Seoul, Korea.

Kim, B.H, K.J. Lee, and J.S., Kim. 2000. Monitoring of plant ecosystem in Gildong ecological park. Korean Soc. Environ. Ecol., Seoul, Korea.

Korea National Arboretum. 2015. Gardener's diary. Seoul, Korea: Geobook.

Korea National Arboretum of Korea Forest Service. 2008. Rare plants data book of Korea. Seoul, Korea: Geobook.

Nature. 2015. Korean plant names index committee. Retrieved from http://www.nature.go.kr/kpni/index.do.

Lee, W.C. 1996. Lineamenta florae koreae. Seoul, Korea: Academy books.

Lee, C.B. 2003. Coloured flora of korea. Seoul, Korea: Hyangmunsa. Nam, C.H., Y.C. Jeon, E.J. Ahn, Y.J. Kim, H.Y. Jin, and C.h. Lee. 2017, February. Occurrence patterns and available offer of weeds in the garden of Korea national arboretum. Korean Forest Society, Seoul, Korea.

Oh, K.A. 2008. Rustic garden. Seoul, Korea: Design house.

Park. S.H. 2009. New illustrations and photographs of naturalized plants of Korea. Seoul, Korea: Ilchokak.

Reu, I.S. 2016. A Study on ecology and environmental education program on naturalized plants for primary school based on naturalized plants research. MS thesis, Korea Natl. Univ. Educ., Cheongju, Korea.

Rural Development Administration National Institute of Agricultural Sciences. 2008. Weed management guide. Anyang, Korea: Agriculture \& Horticulture.

Son, B.D. 2004. The Ecological Administration Plan of World Cup Park by the Monitoring of Ecosystem. MS thesis. Univ. Seoul, Seoul, Korea. 